\title{
Zentrale Schlafapnoe warnt vor Herzinsuffizienz
}

\author{
In einer Studie erwiesen sich zentrale Schlafapnoe und Cheyne-Stokes-Atmung als Risikofaktoren \\ für Herzinsuffizienz. Für obstruktive Apnoe-Formen fand sich dagegen kein Zusammenhang.
}

\begin{abstract}
Für die prospektive Longitudinalstudie wurden 2.865 Männer im Alter von $76,3 \pm 5,5$ Jahre mit einem Body-MassIndex von $27,2 \pm 3,8 \mathrm{~kg} / \mathrm{m}^{2}$ rekrutiert, die bereits in die multizentrischen „Osteoporotic Fractures in Men Study“ eingeschlossen waren [Blank JB et al. Contemp Clin Trials 2005;26:557-68]. Sie erhielten zu Beginn eine unüberwachte Polysomnografie zu Hause. Alle vier Jahre wurden sie postalisch im Hinblick auf neu aufgetretene oder dekompensierte Herzinsuffizienz nachverfolgt, im Mittel 7,3 Jahre lang.

Hauptindikatoren für das Vorliegen einer schlafbezogenen Atmungsstörung waren der obstruktive Apnoe-Hypopnoe-Index (AHI), der zentrale ApnoeIndex (CAI) und eine Cheyne-StokesAtmung. Als kovariate Faktoren wurden Alter, ethnische Zugehörigkeit, klini-
\end{abstract}

scher Status, Komorbiditäten, körperliche Aktivität sowie Alkohol- und Tabakkonsum berücksichtigt.

Ein erhöhter AHI-Wert war nicht mit dem Auftreten einer Herzinsuffizienz vergesellschaftet. Hingegen ergab sich für einen CAI-Wert $\geq 5$ eine angepasste Hazard Ratio (HR) von 1,79 (95\%-Konfidenzintervall: 1,16-2,77), für eine Cheyne-Stokes-Atmung sogar von 2,23 $(1,45-3,43)$. Ohne die Teilnehmer, die bereits zu Beginn an Herzinsuffizienz litten, betrugen die Werte noch 1,57 (0,92-2,66) bzw. 1,90 (1,45-3,43).

- Javaheri S, Blackwell T, Ancoli-Israel S et al. Sleep Disordered Breathing and Incident Heart Failure in Older Men. Am J Respir Crit Care Med. 2016;193:561-8

\section{KOMMENTAR}

Hoch aktuell ist das Thema angesichts der SERVE-HF-Studie [Cowie MR et al. N Engl]
Med. 2015;373:1095-105]. Darin führte die Adaptive Servo-Ventilation (ASV) bei zentraler Schlafapnoe und systolischer Herzinsuffizienz entgegen allen Erwartungen zu einer höheren Mortalität.

Neu ist, dass gerade im höheren Alter schlafbezogene Atmungsstörungen einen Risikofaktor für die Entwicklung einer Herzinsuffizienz darstellen könnten. Es überrascht allerdings, dass offensichtlich nur die sogenannten zentralen, nicht aber die obstruktiven schlafbezogenen Atmungsstörungen Risikofaktoren sind - wobei die Autoren einräumen, dass die Syndrome auch eine Art respiratorische Frühmanifestation der Herzinsuffizienz sein könnten. Die interessante Frage, ob die Atmungsanalyse im Schlaf vielleicht sensitiver zur Früherkennung der Herzinsuffizienz ist als andere klinische Methoden, lässt sich leider noch nicht beantworten.

Prof. Dr. med. K. Rasche

\section{Eine richtig lausige Diagnose}

Ein 56-jähriger Mann litt seit drei Monaten unter einem generalisierten Pruritus. Bei der sorgfältigen körperlichen Untersuchung stellte man klei-

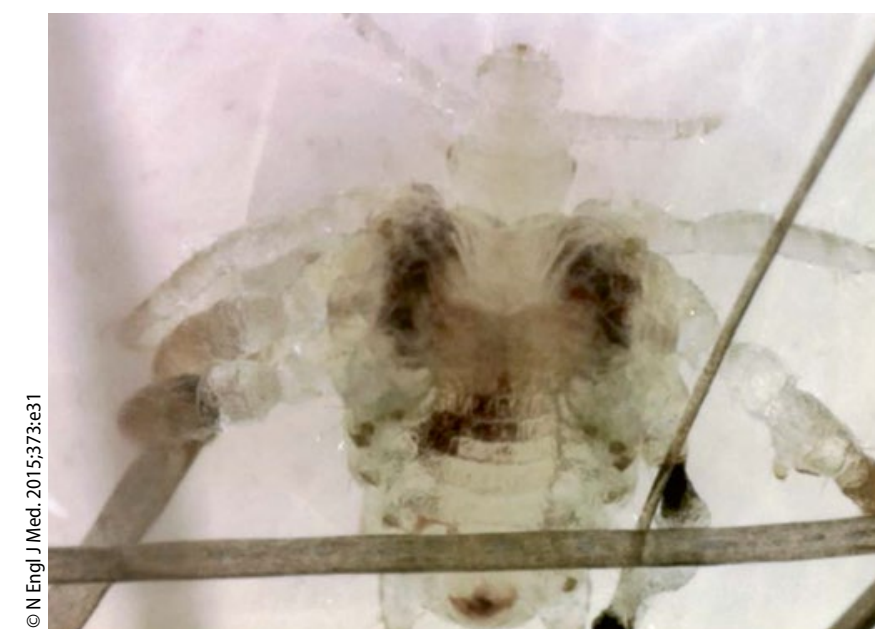

Filzlaus (Pthirus pubis, vulgär "Sackratte“) in der Körperbehaarung, dargestellt per Dermatoskopie. ne schwarze Punkte zwischen den Körperhaaren im Bereich von Abdomen und Becken fest. Mittels Dermatoskopie fand man Filzläuse. Der Patient wurde erfolgreich behandelt durch Rasieren der Haare am Bauch und im Schambereich und mit einer einmaligen achtstündigen Anwendung von 5\%iger Permethrin-Creme.

Die Filzlaus ist mit 1-1,5 mm Länge etwas kleiner als die Kopflaus und weist eine eher runde Form und dicke Hinterbeine mit kräftigen Halteklauen auf. Damit hält sie sich an den Haaren des Menschen fest. An den Haaren werden auch die Eier befestigt, die sogenannten Nissen. Der Stich der Filzlaus verursacht meist einen starken Juckreiz und eine blaue Verfärbung der betroffenen Hautpartie. Filzläuse kommen vor allem in der Schambehaarung, seltener in den Achsel- und Barthaaren und noch seltener in den Wimpern und Augenbrauen vor.

Die Übertragung erfolgt unter schlechten hygienischen Bedingungen und bei engem Körperkontakt, vor allem auch bei Sexualkontakten. Daher zählt der Filzlausbefall zu den sexuell übertragenen Erkrankungen. Die geringe Wertschätzung drückt sich auch in der volkstümlichen Bezeichnung der Tiere aus: Sackratten.

Prof. Dr. med. H. S. FüeßI 